\title{
Anti-Staphylococcal Biofilm Effects of Human Cathelicidin Peptides
}

\author{
Biswajit Mishra, Radha M. Golla, Kyle Lau, Tamara Lushnikova, and Guangshun Wang* \\ Department of Microbiology and Pathology, University of Nebraska Medical Center, 986495 Nebraska Medical Center, Omaha, \\ Nebraska 68198-6495, United States
}

Supporting Information
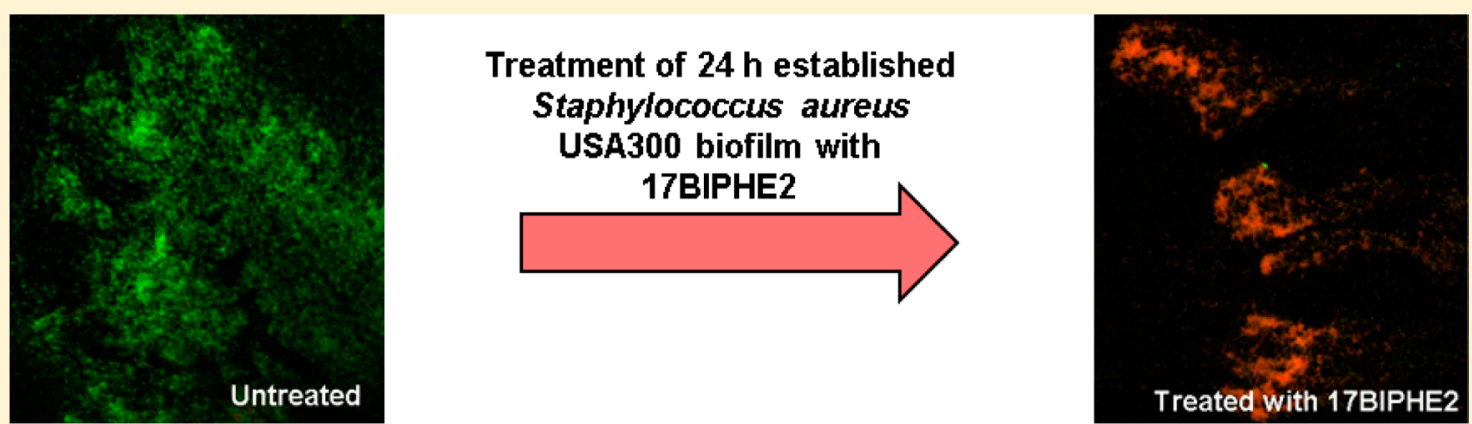

ABSTRACT: Staphylococcus aureus can live together in the form of biofilms to avoid elimination by the host. Thus, a useful strategy to counteract bacterial biofilms is to re-engineer human antimicrobial peptide LL-37 so that it can be used as a remedy for preventing and removing biofilms. This study reports antibiofilm effects of four human cathelicidin LL-37 peptides against community-associated and hospital isolated methicillin-resistant Staphylococcus aureus (MRSA) strains. Although the intact molecule LL-37 inhibited biofilm formation at low concentrations, it did not inhibit bacterial attachment nor disrupt preformed biofilms. However, two 17-residue peptides, GF-17 and 17BIPHE2, inhibited bacterial attachment, biofilm growth, and disrupted established biofilms. An inactive peptide RI-10 was used as a negative control. Our results obtained using the $S$. aureus mutants in a static biofilm model are consistent with the literature obtained in a flow cell biofilm model. Because 17BIPHE2 is the most effective biofilm disruptor with desired stability to proteases, it is a promising lead for developing new anti-MRSA biofilm agents.

KEYWORDS: Biofilms, 17BIPHE2, GF-17, LL-37, attachment mutants, Staphylococcus aureus

Staphylococcus aureus is a dangerous pathogen commonly associated with skin and soft tissue infections involving abscesses, pneumonia, and sepsis. ${ }^{1}$ Resistant Staphylococcal strains (i.e., superbugs) were initially isolated in clinical settings and later from communities. Methicillin-resistant S. aureus (MRSA) is frequently involved in biofilms in wounds and indwelling medical devices. ${ }^{2}$ As a consequence, MRSA became difficult to treat with currently used antibiotics, and new types of anti-Staphylococcal biofilm compounds are of critical importance. 3,4

Like other living organisms, humans possess elegant innate defense systems that ward off infectious pathogens. Over 100 antimicrobial peptides and proteins (AMPs) have been identified from various human tissues. ${ }^{5}$ The major AMPs in humans are cathelicidins, defensins, dermcidins, hepcidins, RNases, and histatins. ${ }^{6}$ Human cathelicidin LL-37 is a wellstudied AMP with activity against bacteria, viruses, fungi, and parasites. In addition, LL-37 can regulate immune response, wound healing, and apoptosis. ${ }^{7-10}$ As a consequence, there is a high interest to engineer novel therapeutic compounds based on this peptide template. Indeed, multiple fragments of human LL-37 have been identified for developing antibacterial, antiviral, and antibiofilm peptides. ${ }^{11}$

The antibiofilm activity of human LL-37 against Pseudomonas aeruginosa was first described in $2008 .^{12}$ LL-37 also inhibits the attachment and biofilm formation of Staphylococcus epidermidis $^{13}$ and S. aureus ${ }^{14}$ at low concentrations. In addition, the LL37 molecule made using D-amino acids is also effective in preventing biofilm formation. ${ }^{14}$ Of note, LL-37 fragments such as P10 and P60.4Ac are more active than LL-37 in preventing biofilm formation of MRSA in thermally wounded human skin equivalents. ${ }^{15}$ Other fragments of LL-37, such as KS-30, KR-20, and KR-12, are inhibitory to the biofilms of multidrug-resistant Acinetobacter baumannii. ${ }^{16}$ Finally, LL-37 and its truncated peptide variants are potent against the biofilms of Burkholderia pseudomallei and $P$. aeruginosa. ${ }^{17-21}$

Most of the studies mentioned above deal with the effects of LL-37 on biofilms at the early stage such as attachment and biofilm growth. It is realized, however, that the later stage of biofilms (e.g., mature or preformed biofilms) is most challenging to treat. ${ }^{22}$ Another limitation is that the native sequences of LL-37 and its fragments utilized in those studies can be rapidly degraded by proteases. To improve peptide stability, we have engineered a series of anti-MRSA compounds, including 17BIPHE2, based on GF-17. This was achieved by

Received: November 12, 2015

Accepted: December 4, 2015

Published: December 4, 2015 
introducing nonstandard amino acids such as D-leucines and biophenylalanines. ${ }^{23}$ Both GF-17 and 17BIPHE2 consist of 17 amino acids owing to the addition of a glycine at the $\mathrm{N}$ terminus. GF-17, with a helical conformation, is the major antimicrobial peptide of human LL-37 corresponding to residues $17-32 . .^{24}$ 17BIPHE2, with a largely nonhelical conformation, is resistant to several proteases such as chymotrypsin, S. aureus protease V8, and fungal proteinase $\mathrm{K}$. In addition, 17BIPHE2 prevents $S$. aureus biofilm formation in a mouse model. ${ }^{23}$ However, there is no study that evaluates the biofilm disruptive ability of 17BIPHE2. A potent biofilm disruptor is needed because it might be utilized to get rid of preformed biofilms in medical devices such as catheters to avoid the painful replacement surgery. In addition, it is not clear to what extent 17BIPHE2 is superior to human LL-37 and its natural fragments in terms of antibiofilm capability. In this study, we compare the antibiofilm capabilities of the engineered peptide 17BIPHE2 with human LL-37, GF-17, and RI-10 against a variety of $S$. aureus clinical strains. An inactive peptide $\mathrm{RI}-10,{ }^{25,26}$ obtained by further truncating GF-17, is included as a negative control. To reduce potential cytotoxicity and the amount of peptide needed for treatment, we also evaluate combined effects of the potent peptides with daptomycin or vancomycin. To provide additional insight, we also evaluate select $S$. aureus transposon mutants that are defective in attachment and maturation genes for biofilm formation. Our study reveals that 17BIPHE2 can disrupt the preformed biofilms of $S$. aureus USA300.

We first compared the antimicrobial activity of these peptides against planktonic $S$. aureus. The minimal inhibitory concentration (MIC) of LL-37 is known to depend on media conditions. ${ }^{10}$ We used $25 \%$ tryptic soy broth (TSB) in our assays since LL-37 is able to kill $S$. aureus USA300 in this media. LL-37 was found to be active against $S$. aureus USA300 and USA400, but not $S$. aureus USA200, Mu50, and UAMS-1 strains at $50 \mu \mathrm{M}$. Thus, antibacterial activity of human LL-37 also depends on the types of $S$. aureus stains (Table 1). GF-17

Table 1. Minimal Inhibitory Concentrations of LL-37 and Its Derivatives against $S$. aureus Clinical Strains ${ }^{a}$

\begin{tabular}{lcccccc} 
& \multicolumn{6}{c}{ MIC $(\mu \mathrm{M})$} \\
\cline { 2 - 7 } peptide & USA200 & USA300 & USA400 & MU50 & UAMS-1 & Newman \\
LL-37 & $>50$ & 12.5 & 6.25 & $>50$ & $>50$ & $>50$ \\
GF-17 & 1.56 & 1.56 & 1.56 & 1.56 & 3.1 & 1.56 \\
17BIPHE2 & 3.1 & 3.1 & 3.1 & 3.1 & 3.1 & 3.1 \\
RI-10 & $>50$ & $>50$ & $>50$ & $>50$ & $>50$ & $>50$
\end{tabular}

${ }^{a}$ Bactericidal assays were done using $25 \%$ TSB.

and 17BIPHE2 are more potent than their parent peptide LL37 against all of these Staphylococcal strains (MIC 1.56-3.1 $\mu \mathrm{M})$. RI-10 was previously found to be inactive against $E$. coli or S. aureus. ${ }^{25,26}$ It is not surprising that this 10-residue peptide showed no activity against these clinically important $S$. aureus strains (MIC > $50 \mu \mathrm{M})$ (Table 1$)$.

We then evaluated the antibiofilm potency of these LL-37 peptides based on three types of experiments in 96-well polystyrene microplates. ${ }^{3}$ The first experiment evaluates to what extent the peptide can inhibit bacterial attachment to the surface. Surface attachment is regarded as the first step for biofilm formation. The results are shown in Figure 1. In the concentration range from 3.1 to $25 \mu \mathrm{M}$, there is a trend of biomass decrease with increase in peptide concentration,

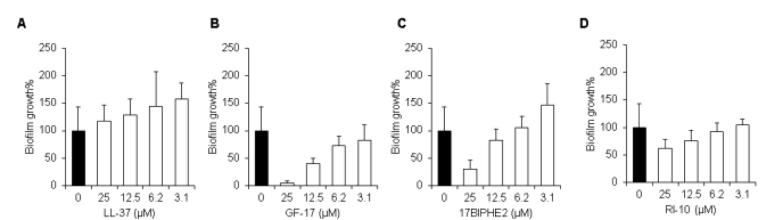

Figure 1. Inhibition of the attachment of $S$. aureus USA300 by LL-37 (A), GF-17 (B), 17BIPHE2 (C), and RI-10 (D).

especially for GF-17 and 17BIPHE2, which were able to inhibit the attachment almost entirely at $25 \mu \mathrm{M}$. In contrast, RI10 only showed a very small effect at $25 \mu \mathrm{M}$, and LL-37 was unable to inhibit the attachment of $S$. aureus USA300.

The second experiment was designed to assess the extent of biofilm inhibition by these LL-37 peptides. The results are presented in Figure 2. Consistent with the literature, ${ }^{13,14}$ LL-37

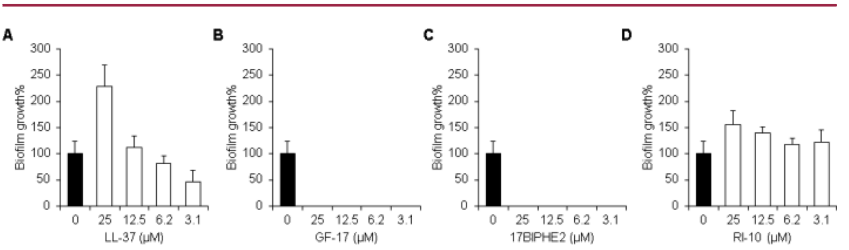

Figure 2. Inhibition of biofilm growth of S. aureus USA300 by LL-37 (A), GF-17 (B), 17BIPHE2 (C), and RI-10 (D).

inhibited about $50 \%$ biofilm formation of S. aureus USA300 only at a low concentration of $3.1 \mu \mathrm{M}$, as there was an increase in biofilm mass with the increase in peptide concentration from 6.2 to $25 \mu \mathrm{M}$ (panel A). This is likely due to LL-37 oligomerization at higher concentrations ${ }^{10}$ that could facilitate the binding of bacterial extracellular DNA (eDNA), an important component involved in biofilms, thereby increasing the amount of biofilm biomass. In the entire concentration range (3.1-25 $\mu \mathrm{M}), \mathrm{GF}-17$ and 17BIPHE2 completely inhibited the growth of the $S$. aureus biofilms (panels B and C). On the contrary, RI-10 was unable to inhibit biofilm growth (panel D).

In the third experiment, we tested the power of these LL-37 peptides in destroying established biofilms of several clinical strains of $S$. aureus. At elevated concentrations $(12.5-25 \mu \mathrm{M})$, 17BIPHE2 could remove the biofilms of all the staphylococcal strains, including USA200, USA300, USA400, Mu50, Newman, and UAMS-1 (Figure 3C,G,K,O,S,W). GF-17 was also highly potent against those strains (panels B, F, J, N, and R) except for S. aureus UAMS-1 (panel V). At $12.5 \mu \mathrm{M}$, S. aureus USA400 appeared to be slightly more difficult to remove by GF-17 or 17BIPHE2 than either $S$. aureus USA200 or S. aureus USA300. However, neither LL-37 (panels A, E, I, M, Q and U) nor RI10 (panels D, H, L, P, T, and X) was effective in biofilm disruption. To provide direct evidence for live and dead cells in the $S$. aureus USA300 biofilms, we utilized confocal microscopy. The live cells without treatment are in green (Figure 4A), while the dead cells are in red as a result of treatment with GF-17 (panel B) or 17BIPHE2 (panel C). It is thus established that GF-17 and 17BIPHE2 did kill S. aureus even in mature biofilms (24 h old).

To validate our static biofilm model and provide additional insight into biofilm physiology, we selected four important mutants (icaA, $f n b A$, altA, and sasC) from the Nebraska Transposon Mutant Library. ${ }^{27}$ Each of these mutants contains a disrupted nonessential gene. These four $S$. aureus mutants are 


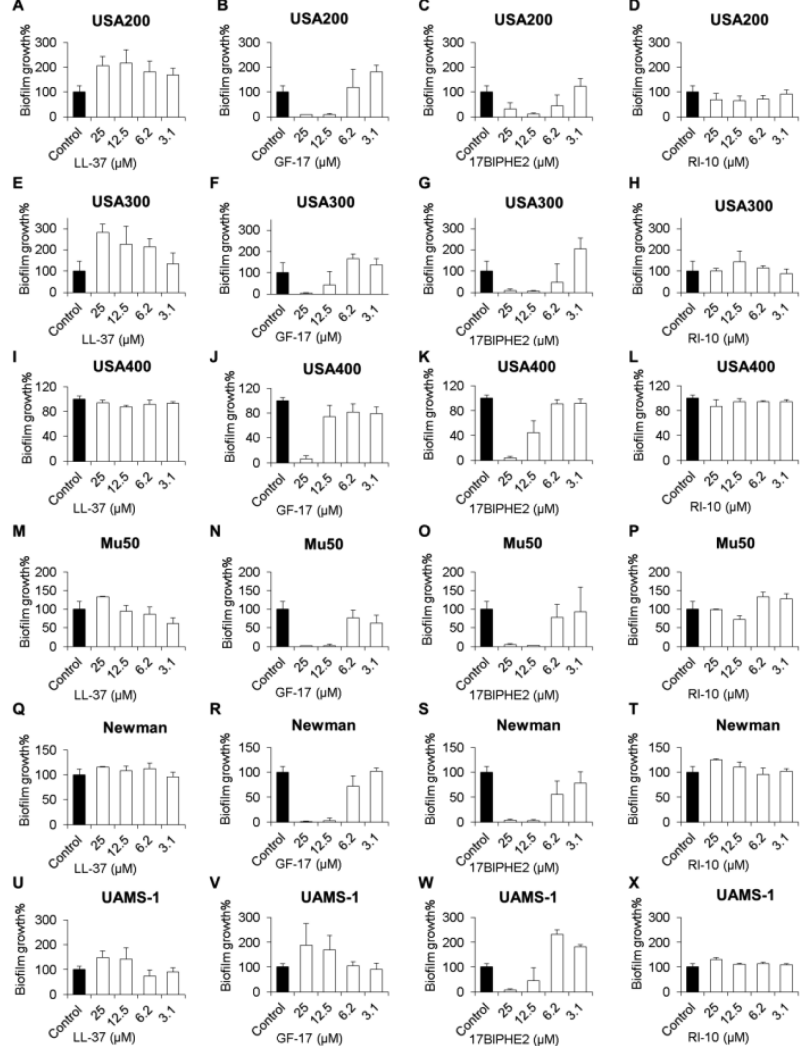

Figure 3. Disruption of the $24 \mathrm{~h}$ preformed biofilms of $S$. aureus clinical strains by LL-37 (A, E, I, M, Q and U), GF-17 (B, F, J, N, R, and V), 17BIPHE2 (C, G, K, O, S, and W), and RI-10 (D, H, L, P, T, and X). The clinical strains include S. aureus USA200 (A-D), USA300 $(\mathrm{E}-\mathrm{H})$, USA400 (I-L), Mu50 (M-P), Newman (Q-T), and UAMS$1(\mathrm{U}-\mathrm{X})$.

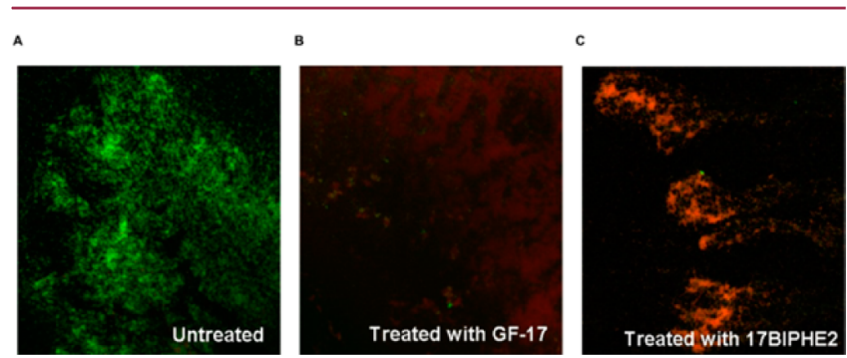

Figure 4. Confocal microscopic images of the $24 \mathrm{~h}$ biofilms of $S$. aureus USA300 for (A) untreated, (B) treated with GF-17, and (C) treated with 17BIPHE2. Biofilms were stained with the live (green) and dead (red) stain kit, which uses the SYTP-9 dye to stain live cells and propidium iodide (PI) to stain dead cells.

well characterized to affect biofilm attachment and maturation. $^{28,29}$ While $S$. aureus biofilm slime layer is composed of teichoic acid (80\%) and host cell proteins, a specific polysaccharide intracellular antigen (PIA) is used by many staphylococcus species. Of note, USA300 clones or JE2 (a USA300 LAC derivative) biofilms are shown to be independent of PIA based matrix. ${ }^{28}$ We selected the icaA biosynthetic gene mutant that involves the icaR regulatory element to carry out the PIA-dependent biofilm formation. Indeed, no defects in initial attachment or in the $24 \mathrm{~h}$ matured biofilms were observed for this mutant (Figure 5A,G). Furthermore, the 17BIPHE2 treatment affects the initial attachment and disrupts the established biofilms of JE2 (wild type in the transposon

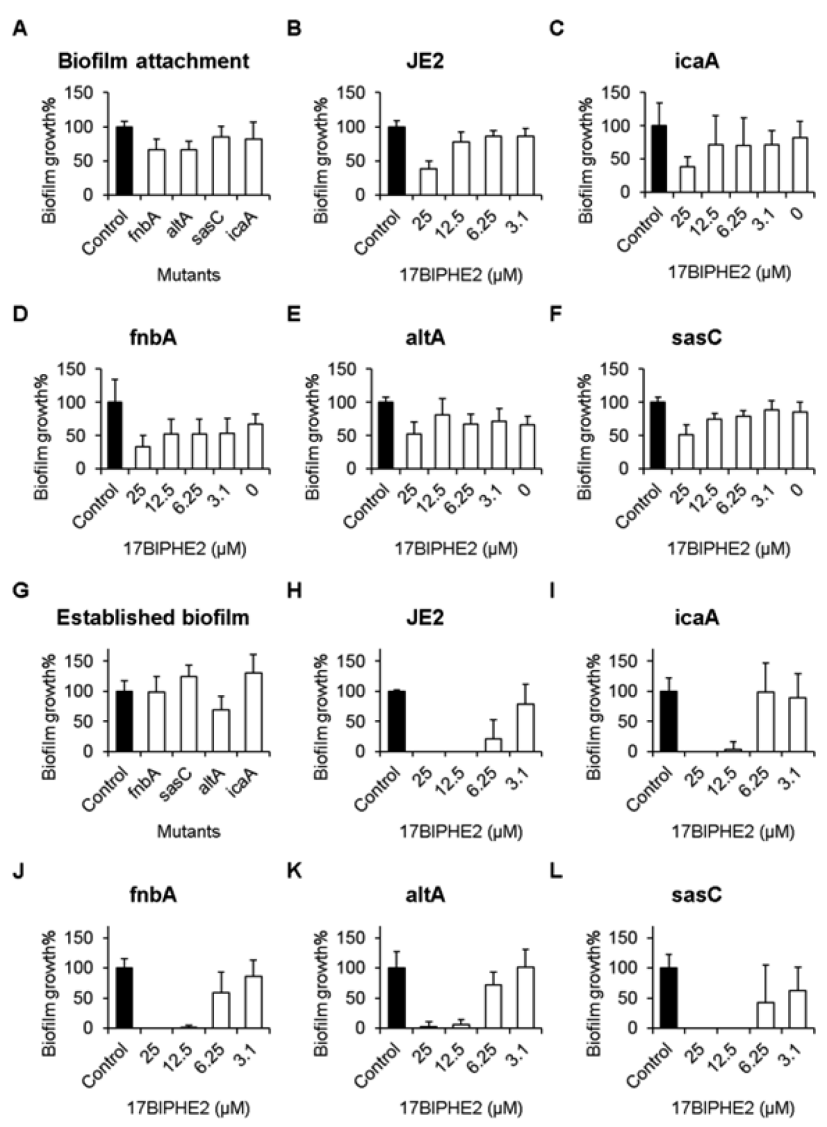

Figure 5. Attachment and biofilm disruption of various transposon mutants of $S$. aureus. This figure evaluates the $S$. aureus USA300 mutant with and without treatment with 17BIPHE2 on initial attachment of the wild type JE2 (A, G) and mutants (B, C, D, E and F), as well as on the established biofilms (H, I, J, K and L) of JE2 (control), icaA, $f n b A$, altA, and sasC, respectively.

library) (Figure 5C,I) in a fashion similar to S. aureus USA300 (Figures 1C and 3G). In contrast, the PIA-independent pathway involves microbial surface components that recognize adhesive matrix molecules (MSCRAMM), such as fibronectinbinding proteins FnbA and FnbB, elastin-binding protein, clumping factor $\mathrm{A}$ and $\mathrm{B} .^{28}$ We selected the $f n b A$ mutant and found reduced biofilm attachment (Figure 5A). Moreover, the 24-h matured biofilm was similar to the wild type (Figure 5G). Treatment of $f n b A$ with 17BIPHE2 showed reduction in attachment and disruption similar to the wild type JE2 (Figure $5 \mathrm{D}, \mathrm{J})$. The altA gene (autolysin) is regulated by the $f_{n b P}$ gene. ${ }^{30}$ Unlike the $f n b A$ mutant, the alt $A$ mutant showed reduced biomass in the mature biofilm, consistent with a recent biofilm observation made in a BioFlux system. ${ }^{28}$ Moreover, this mutant showed a similar trend to the $f n b A$ mutant with and without 17BIPHE2 treatment (Figure 5E,K). We also included $s a s C$, a cell surface anchor protein actively involved in biofilm attachment and maturation. ${ }^{31}$ This transposon mutant, with (Figure 5F,L) and without treatment (Figure 5A,G), behaved the same as the wild type in bacterial attachment or biofilm formation. Overall, our results from the static model essentially agree with those obtained from the fluidic BioFlux system. ${ }^{28}$ Other studies also support the tendencies that $S$. aureus biofilm matrices are less dependent on cell surface protein but rely more on cytoplasmic proteins associated with the stationary growth phase. ${ }^{32}$ Although both the $f n b A$ and alt $A$ mutants are defective in biofilm attachment, only altA showed a reduced 
biomass in the 24-h mature biofilms (Figure 5A,G). Interestingly, the treatment of all the mutants with 17BIPHE2 led to biofilm masses equal to the wild type, implying these genes are insignificant in response to the peptide.

In conclusion, AMPs are effective antibiofilm compounds, ${ }^{22,33}$ and human cathelicidin LL-37 has been established as an important antibiofilm peptide. ${ }^{12-21}$ As an advance, we have recently engineered LL-37 into a potent, selective, and stable peptide. ${ }^{23}$ This study compared antibiofilm activities of this engineered peptide 17BIPHE2 with LL-37 and its two native fragments. Consistent with the literature, ${ }^{15,20}$ LL-37 was found to inhibit biofilm growth of $S$. aureus USA300 in vitro only at a low concentration. We also observed that LL-37 was unable to inhibit bacterial attachment or disrupt preformed biofilms. Although they have different conformations, ${ }^{24,34}$ both 17BIPHE2 and GF-17 displayed potent antibiofilm activity. They can inhibit not only bacterial attachment (Figure 1) but also biofilm growth of these strains (Figure 2). Remarkably, 17BIPHE2 and GF-17 can also disrupt the 24-h formed biofilms (Figures 3 and 4). Thus, both peptides have antibiofilm potency superior to their parent molecule LL-37. In addition, both peptides at $6.2 \mu \mathrm{M}$ can disrupt even 72 -h preformed biofilms of $S$. aureus USA300 in the presence of 1.6 $\mu \mathrm{M}$ of daptomycin or vancomycin (Figure 6), opening the door

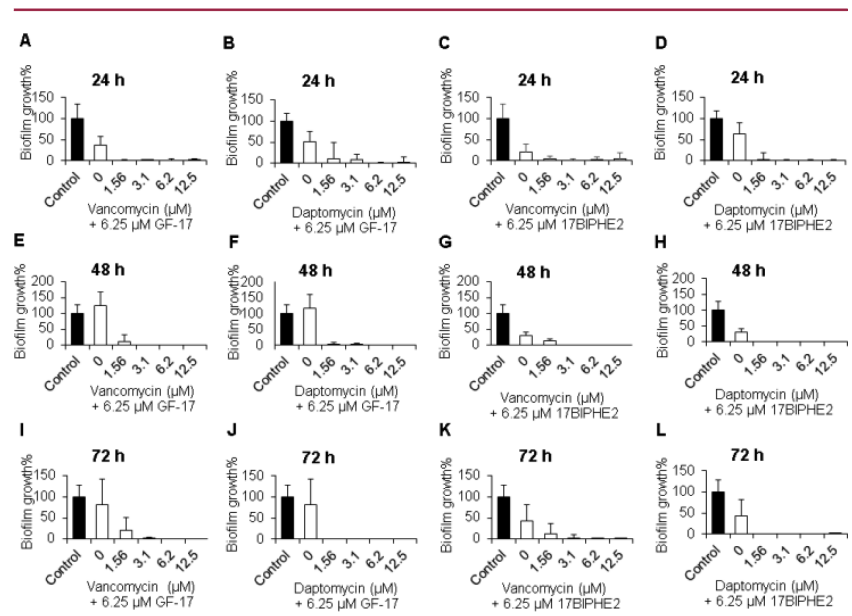

Figure 6. Combination treatment of the peptide with existing antibiotics on the $S$. aureus USA300 biofilms established in 24, 48, and $72 \mathrm{~h}$. Panels A, E, and I are the combination of GF-17 and vancomycin; panels $\mathrm{B}, \mathrm{F}$, and $\mathrm{J}$ are the combination of GF-17 and daptomycin; panels $\mathrm{C}, \mathrm{G}$, and $\mathrm{K}$ are the combination of 17BIPHE2 and vancomycin; and panels $\mathrm{D}, \mathrm{H}$, and $\mathrm{L}$ are the combination of 17BIPHE2 and daptomycin.

to potential combination therapy. Combined treatments can reduce the concentration of peptide, thereby reducing both potential cytotoxicity and peptide cost. Indeed, both GF-17 and 17BIPHE2 showed low cytotoxicity (50\% hemolytic concentrations, $\sim 120 \mu \mathrm{M}$ ) (Supporting Information, Figure S1). However, 17BIPHE2 possesses some advantages over GF-17. 17BIPHE2 is potent against the preformed biofilms of $S$. aureus UAMS-1 (Figure 3) and can work even in the presence of proteases such as chymotrypsin and $S$. aureus protease $\mathrm{V} 8 .^{23}$ In contrast, GF-17 is unable to do so. Taken together, 17BIPHE2 with both in vitro (this study) and in vivo ${ }^{23}$ antibiofilm activity can be a promising lead for developing novel antibiofilm agents.
Our inclusion of a panel of $S$. aureus single-gene mutants achieved two goals. First, it validated our static biofilm model because our results (Figure 5) are fully consistent with the literature using the flow cell model. ${ }^{28}$ Second, it sheds light on the genetic basis of biofilm formation. Since we obtained similar results using these mutants (Figure 5), such staphylococcal genes are unimportant in response to the action of 17BIPHE2. Future identification of the response genes of $S$. aureus USA300 to the engineered peptide 17BIPHE2 will not only enhance our knowledge on bacterial resistance mechanisms but also inspire the design of better antimicrobials in urgent need.

\section{ASSOCIATED CONTENT}

\section{S Supporting Information}

The Supporting Information is available free of charge on the ACS Publications website at DOI: 10.1021/acsmedchemlett.5b00433.

Experimental procedures and one figure for peptide cytotoxicity (PDF)

\section{AUTHOR INFORMATION}

\section{Corresponding Author}

*E-mail: gwang@unmc.edu.

\section{Author Contributions}

G.W. conceived the project. All the authors conducted the experiments and analyzed the data. G.W. and B.M. wrote the paper. All the authors have given approval to the final version of the manuscript.

\section{Funding}

This study was supported by the NIAID/NIH grant R01AI105147 to GW.

\section{Notes}

The authors declare no competing financial interest.

\section{ACKNOWLEDGMENTS}

The authors thank Kenneth W. Bayles for providing us a copy of the Nebraska Transposon Mutant Library for Staphylococcus aureus USA300, and Janice Taylor and James R. Talaska for assistance with confocal microscopy.

\section{ABBREVIATIONS}

AMP, antimicrobial peptide; 17BIPHE2, 17-residue biphenylalanine-containing anti-MRSA peptide designed based on GF17; GF-17, peptide corresponding to the major antimicrobial region of LL-37; LL-37, human cathelicidin gene-coded peptide with 37 amino acids; MIC, minimal inhibitory concentration; MRSA, methicillin-resistant Staphylococcal aureus; RI-10, LL-37 derived peptide corresponding to residues 19-28

\section{REFERENCES}

(1) David, M. Z.; Daum, R. S. Community-associated methicillinresistant Staphylococcus aureus: epidemiology and clinical consequences of an emerging epidemic. Clin. Microbiol. Rev. 2010, 23, 616-687.

(2) Otto, M. Staphylococcal biofilms. Curr. Top. Microbiol. Immunol. 2008, 322, 207-228.

(3) Mishra, B.; Lushnikova, T.; Wang, G. Small lipopeptides possess antibiofilm capability comparable to daptomycin and vancomycin. RSC Adv. 2015, 5, 59758-59769.

(4) Chen, M.; Yu, Q.; Sun, H. Novel strategies for the prevention and treatment of biofilm related infections. Int. J. Mol. Sci. 2013, 14, 18488-18501. 
(5) Wang, G.; Li, X.; Wang, Z. APD2: the updated antimicrobial peptide database and its application in peptide design. Nucleic Acids Res. 2009, 37, D933-7.

(6) Wang, G. Human antimicrobial peptides and proteins. Pharmaceuticals 2014, 7, 545-594.

(7) Wang, G. Antimicrobial Peptides: Discovery. In Design and Novel Therapeutic Strategies; CABI: Oxfordshire, U.K., 2010.

(8) Cederlund, A.; Gudmundsson, G. H.; Agerberth, B. Antimicrobial peptides important in innate immunity. FEBS J. 2011, 278, 39423951.

(9) Vandamme, D.; Landuyt, B.; Luyten, W.; Schoofs, L. A comprehensive summary of LL-37, the factotum human cathelicidin peptide. Cell. Immunol. 2012, 280, 22-35.

(10) Lehrer, R. I.; Ganz, T. Cathelicidins: a family of endogenous antimicrobial peptides. Curr. Opin. Hematol. 2002, 9, 18-22.

(11) Wang, G.; Mishra, B.; Epand, R. F.; Epand, R. M. High-quality 3D structures shine light on antibacterial, antibiofilm and antiviral activities of human cathelicidin LL-37 and its fragments. Biochim. Biophys. Acta, Biomembr. 2014, 1838, 2160-2172.

(12) Overhage, J.; Campisano, A.; Bains, M.; Torfs, E. C.; Rehm, B. H.; Hancock, R. E. Human host defense peptide LL-37 prevents bacterial biofilm formation. Infect. Immun. 2008, 76, 4176-4182.

(13) Hell, E.; Giske, C. G.; Nelson, A.; Romling, U.; Marchini, G. Human cathelicidin peptide LL37 inhibits both attachment capability and biofilm formation of Staphylococcus epidermidis. Lett. Appl. Microbiol. 2010, 50, 211-215.

(14) Dean, S. N.; Bishop, B. M.; van Hoek, M. L. Natural and synthetic cathelicidin peptides with anti-microbial and antibiofilm activity against Staphylococcus aureus. BMC Microbiol. 2011, 11, 1142180-11-114.

(15) Haisma, E. M.; de Breij, A.; Chan, H.; van Dissel, J. T.; Drijfhout, J. W.; Hiemstra, P. S.; El Ghalbzouri, A.; Nibbering, P. H. LL-37-derived peptides eradicate multidrug-resistant Staphylococcus aureus from thermally wounded human skin equivalents. Antimicrob. Agents Chemother. 2014, 58, 4411-4419.

(16) Feng, X.; Sambanthamoorthy, K.; Palys, T.; Paranavitana, C. The human antimicrobial peptide LL-37 and its fragments possess both antimicrobial and antibiofilm activities against multidrug-resistant Acinetobacter baumannii. Peptides 2013, 49, 131-137.

(17) Dosler, S.; Karaaslan, E. Inhibition and destruction of Pseudomonas aeruginosa biofilms by antibiotics and antimicrobial peptides. Peptides 2014, 62, 32-37.

(18) Dean, S. N.; Walsh, C.; Goodman, H.; van Hoek, M. L. Analysis of mixed biofilm (Staphylococcus aureus and Pseudomonas aeruginosa) by laser ablation electrospray ionization mass spectrometry. Biofouling 2015, 31, 151-161.

(19) Wnorowska, U.; Niemirowicz, K.; Myint, M.; Diamond, S. L.; Wroblewska, M.; Savage, P. B.; Janmey, P. A.; Bucki, R. Bactericidal activities of cathelicidin LL-37 and select cationic lipids against the hypervirulent Pseudomonas aeruginosa strain LESB58. Antimicrob. Agents Chemother. 2015, 59, 3808-3815.

(20) Dean, S. N.; Bishop, B. M.; van Hoek, M. L. Susceptibility of Pseudomonas aeruginosa Biofilm to Alpha-Helical Peptides: Denantiomer of LL-37. Front. Microbiol. 2011, 2, 128.

(21) Pompilio, A.; Scocchi, M.; Pomponio, S.; Guida, F.; Di Primio, A.; Fiscarelli, E.; Gennaro, R.; Di Bonaventura, G. Antibacterial and antibiofilm effects of cathelicidin peptides against pathogens isolated from cystic fibrosis patients. Peptides 2011, 32, 1807-1814.

(22) Di Luca, M.; Maccari, G.; Maisetta, G.; Batoni, G. BaAMPs: the database of biofilm-active antimicrobial peptides. Biofouling 2015, 31, 193-199.

(23) Wang, G.; Hanke, M. L.; Mishra, B.; Lushnikova, T.; Heim, C. E.; Chittezham Thomas, V.; Bayles, K. W.; Kielian, T. Transformation of human cathelicidin LL-37 into selective, stable, and potent antimicrobial compounds. ACS Chem. Biol. 2014, 9, 1997-2002.

(24) Li, X.; Li, Y.; Han, H.; Miller, D. W.; Wang, G. Solution structures of human LL-37 fragments and NMR-based identification of a minimal membrane-targeting antimicrobial and anticancer region. $J$. Am. Chem. Soc. 2006, 128, 5776-5785.
(25) Wang, G. Structures of human host defense cathelicidin LL-37 and its smallest antimicrobial peptide KR-12 in lipid micelles. J. Biol. Chem. 2008, 283, 32637-32643.

(26) Epand, R. F.; Wang, G.; Berno, B.; Epand, R. M. Lipid segregation explains selective toxicity of a series of fragments derived from the human cathelicidin LL-37. Antimicrob. Agents Chemother. 2009, 53, 3705-3714.

(27) Fey, P. D.; Endres, J. L.; Yajjala, V. K.; Widhelm, T. J.; Boissy, R. J.; Bose, J. L.; Bayles, K. W. A genetic resource for rapid and comprehensive phenotype screening of nonessential Staphylococcus aureus genes. $m$ Bio 2013, 4, e00537-12.

(28) Moormeier, D. E.; Bose, J. L.; Horswill, A. R.; Bayles, K. W. Temporal and stochastic control of Staphylococcus aureus biofilm development. mBio 2014, 5, e01341-14.

(29) Archer, N. K.; Mazaitis, M. J.; Costerton, J. W.; Leid, J. G.; Powers, M. E.; Shirtliff, M. E. Staphylococcus aureus biofilms: properties, regulation, and roles in human disease. Virulence. 2011, $2,445-459$

(30) Houston, P.; Rowe, S. E.; Pozzi, C.; Waters, E. M.; O'Gara, J. P. Essential role for the major autolysin in the fibronectin-binding protein-mediated Staphylococcus aureus biofilm phenotype. Infect. Immun. 2011, 79, 1153-1165.

(31) Foster, T. J.; Hook, M. Surface protein adhesins of Staphylococcus aureus. Trends Microbiol. 1998, 6, 484-488.

(32) Foulston, L.; Elsholz, A. K.; DeFrancesco, A. S.; Losick, R. The extracellular matrix of Staphylococcus aureus biofilms comprises cytoplasmic proteins that associate with the cell surface in response to decreasing $\mathrm{pH}$. mBio 2014, 5, e01667-14.

(33) Wang, G.; Mishra, B.; Lau, K.; Lushnikova, T.; Golla, R.; Wang, $\mathrm{X}$. Antimicrobial peptides in 2014. Pharmaceuticals 2015, 8, 123-150.

(34) Wang, G.; Epand, R. F.; Mishra, B.; Lushnikova, T.; Thomas, V. C.; Bayles, K. W.; Epand, R. M. Decoding the functional roles of cationic side chains of the major antimicrobial region of human cathelicidin LL-37. Antimicrob. Agents Chemother. 2012, 56, 845-856. 